\title{
Spatial beam cleaning in quadratic nonlinear medium
}

\author{
K. Krupa ${ }^{1}$, R. Fona ${ }^{1}$, A. Tonello ${ }^{2}$, A. Labruyère ${ }^{2}$, B. M. Shalaby ${ }^{2,3}$, \\ S. Wabnitz ${ }^{1,4}$, F. Baronio ${ }^{1}$, A. B. Aceves ${ }^{5}$, G. Millot $^{6}$, V. Couderc ${ }^{2}$ \\ ${ }^{1}$ Dipartimento di Ingegneria dell'Informazione, Università di Brescia, via Branze 38, 25123, Brescia, Italy \\ ${ }^{2}$ Université de Limoges, XLIM, UMR CNRS 7252, 123 Av. A. Thomas, 87060 Limoges, France \\ ${ }^{3}$ Physics Department, Faculty of Science, Tanta University, Tanta, Egypt \\ ${ }^{4}$ Istituto Nazionale di Ottica del Consiglio Nazionale delle Ricerche (INO-CNR), Via Branze 45, 25123 Brescia, Italy \\ ${ }^{5}$ Department of Mathematics, Southern Methodist University, Dallas, Texas 75275-0156, USA \\ ${ }^{6}$ Université de Bourgogne Franche-Comté, ICB, UMR CNRS 6303, 9 Av. A. Savary, 21078 Dijon, France
}

katarzyna.krupa@unibs.it

\begin{abstract}
We show experimentally that a laser beam scrambled by propagation in a short segment of multimode fiber may be cleaned by the nonlinear propagation in KTP cristal with type-II second-harmonic generation. (c) 2018 The Author(s)
\end{abstract}

OCIS codes: $190.2620,190.5940,190.4420$.

\section{Introduction}

The multimodal nature of speckled beams can unveil surprising effects in the presence of nonlinearity. Spatial beam Kerr cleaning was recently reported in multimode fibers [1]. Quadratic nonlinearities may offer mechanisms of beam reshaping, and transformations of input elliptical beams into cylindrically symmetric beams have been already illustrated. [2]. Wide input beams and strong quadratic interactions may also lead to the formation of stable polychromatic filaments [3]. In this work we show experimentally that spatial beam self-cleaning may be obtained with type-II KTP crystal cut for second harmonic generation (SHG) operating in the strong conversion regime.

\section{Experimental and numerical results}

In our experiments, we used a high-energy Q-switched mode-locked Nd:YAG laser with $20 \mathrm{~Hz}$ repetition rate emitting $30 \mathrm{ps}$ pulses at $1064 \mathrm{~nm}$. Figure 1 (a) illustrates the structure of our experimental setup. The laser beam is linearly polarized and its orientation was adjusted to optimize the type-II SHG, which took place in a $\mathrm{L}=30 \mathrm{~mm}$ long potassium titanyl phosphate (KTP) crystal. The laser beam before impinging the input facet of the crystal was pre-distorted by a
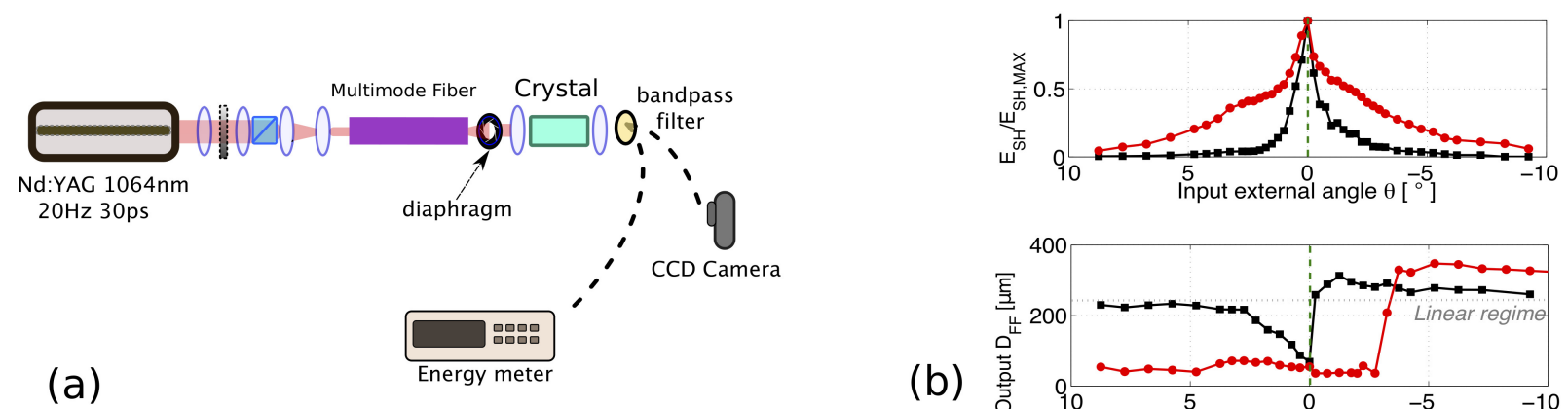

Fig. 1. (a): Experimental setup. (b): experimental results of SHG. KTP crystal at low $0.13 \mathrm{GW} / \mathrm{cm}^{2}$ (black curve) and high $0.9 \mathrm{GW} / \mathrm{cm}^{2}$ (red curve) pump intensities.

10-cm long multimode fiber (MMF) segment with a diameter of $1 \mathrm{~mm}$ and composed by a glass core surrounded by a polymer cladding. The laser beam was reduced and collimated at a diameter of $900 \mu \mathrm{m}$ and coupled into the MMF by two lenses. Since the beam at the output of the MMF was presenting a strong divergence, we put a diaphragm followed 
by a lens of $\mathrm{f}=75 \mathrm{~mm}$ to collimate part of that beam into the KTP crystal. Finally after the crystal we placed a lens to magnify by a factor of four the image, in order to permit beam observation with the camera. A bandpass filter was used to define the spectral window at the fundamental or at the second harmonic, respectively.

We first characterised the SHG conversion efficiency by removing the MMF, and directly coupling the laser beam into the crystal. Fig.1 (b) shows our experimental results for SHG energy and output beam diameter at the fundamental wavelength for different values of the phase mismatch, as obtained by tuning the crystal with different input angles $\theta$. Note that the input beam diameter does not verify the condition of a diffraction length $L_{D}$ being smaller than the crystal length, which is often the case for the direct excitation of spatial solitons $\left(L_{D}<L\right)[5,6]$. We found that at high energy levels the acceptance bandwidth of SHG was broadened, in agreement with the predictions of Ref. [4] for the strong conversion regime. Note also that the self-defocusing effect observed at small negative input angles (from 0 to $-3^{\circ}$ ) at $0.13 \mathrm{GW} / \mathrm{cm}^{2}$ turned into a self-focusing effect when the intensity was increased up to $0.9 \mathrm{GW} / \mathrm{cm}^{2}$, leading to the formation of a self-confined beam. This spatial effect modifies the relative efficiency of SHG upon mismatch, since self-focalised beams at the fundamental lead to larger SHG efficiencies. Indeed, we observed in experiments and in modeling the build up of a progressive asymmetry in SHG efficiency between positive and negative angles $\theta$ when increasing the pump energy. Such asymmetry was even more evident in other types of crystals (here not shown).
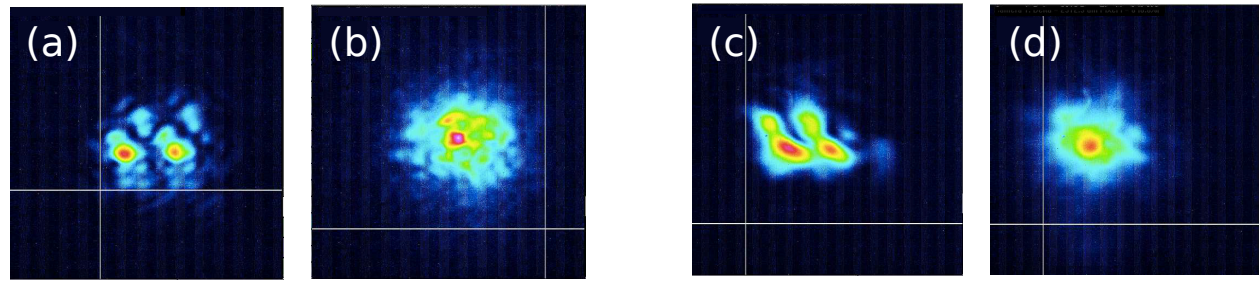

Fig. 2. Fundamental frequency beam at low energy $0.06 \mu J$ (a),(c) and high energy $0.25 \mathrm{~mJ}(\mathrm{~b}),(\mathrm{d})$. Input angle $\theta=1^{\circ}$ (focusing regime (a),(b)), and $\theta=-1^{\circ}$ (defocusing regime, (c),(d)).

The insertion of a MMF in the setup brought a strong distortion to the laser beam, and it was clearly visible by the speckled pattern at the output of the KTP crystal. This fact is illustrated for the fundamental wavelength in Fig.2(a) and (c) in a quasi-linear regime. The two replica of speckles that are well visible in the image are due to the spatial walk-off of the KTP crystal. Surprisingly, when the fundamental beam energy was increased up to $0.25 \mathrm{~mJ}$, we observed the beginning of a beam cleaning effect. The two dominant spots merged in one, as shown by panels (b) and (d). We observed beam self-cleaning up to an angle $\theta=3.5^{\circ}$. Similar dynamics was also found for those small negative angles (defocusing) where high energies bring self-focusing. Panels (c),(d) refer to the case of $\theta=-1^{\circ}$.

In conclusion, we have studied experimentally the nonlinear conversion regime, and how a beam initially distorted by a segment of thick MMF can be cleaned in a KTP crystal with type-II second harmonic generation.

We acknowledge support from: the European Research Council (ERC) under the European Union's Horizon 2020 research and innovation programme, grant No. 740355 and Marie-Sklodowska-Curie grant No. GA-2015-713694 (MULTIPLY); Horiba Medical and Leukos (MSCA MULTIPLY, BECLEAN project); iXcore research foundation; Labex ACTION program (contract ANR-11-LABX-0001-01).

\section{References}

1. K.Krupa, A.Tonello, B.M.Shalaby, M.Fabert, A.Barthélémy, G. Millot, S. Wabnitz, and V. Couderc, "Spatial beam self-cleaning in multimode fibre," Nat. Photon. 11, 234-241 (2017).

2. R. A. Fuerst, B. L. Lawrence, W. E. Torruellas, and G. I. Stegeman, "Beam reshaping by use of spatial solitons in the quadratic nonlinear medium KTP," Opt. Lett. 22, 19-21 (1997).

3. K. Krupa, A. Labruyère, A. Tonello, B. M. Shalaby, V. Couderc, F. Baronio, and A. B. Aceves, "Polychromatic filament in quadratic media: spatial and spectral shaping of light in crystals," Optica 2, 1058-1064 (2015).

4. S. Trillo, S. Wabnitz, R. Chisari, and G. Cappellini,"Two-wave mixing in a quadratic nonlinear medium: bifurcations, spatial instabilities, and chaos,” Opt. Lett. 17, 637-639 (1992).

5. W. E. Torruellas, Z. Wang, E. W. Van Stryland, G. I. Stegeman L. Torner, C. R. Menyuk, "Observation of two-dimensional spatial solitary waves in a quadratic medium," Phys. Rev. Lett. 74, 5036-5040 (1995).

6. B. Bourliaguet, V. Couderc. A. Barthélémy, G. W. Ross, P. G. R. Smith, D. C. Hanna, C. De Angelis, “Observation of quadratic spatial solitons in periodically poled lithium niobate," Opt. Lett. 24, 1410-1412 (1999). 